early phase (0-30s) than G2 ((figure 2A,2B) showed homogeneously iso-enhancement in the early phase) and G3 ((figure $3 \mathrm{~A}, 3 \mathrm{~B})$ showed inhomogeneously iso-enhancement in the early phase) tumors $(p=0.006, p<0.001)$. Besides, the incidence rate of hyper-enhancement in the early phase was higher in G2 tumors than G3 tumors $(p=0.009)$. While in the late phase (31-120s), the incidence rate of iso-enhancement in G1 tumor ((figure 1C) showed iso-enhancement in the late phase) was higher than G2 ((figure 2C) showed hypo-enhancement in the late phase) and G3 ((figure 3C) showed hypo-enhancement in the late phase $)$ tumors $(p=0.001, p<0.001)$. Heterogeneous enhancement and non-enhanced area appeared more often in G2 tumor than G1 tumor $(\mathrm{p}=0.007, \mathrm{p}=0.002)$.

Conclusions Sonographic features, such as tumor size, pancreatic duct dilatation or hepatic metastasis as well as enhancement pattern in each phase may help to predict different pathologic grade of pNETs.

\section{IDDF2020-ABS-0042 HYPERTROPHIC CECAL TUBERCULOSIS: A CASE REPORT}

Marc Ryan Pascua*. De La Salle University Medical Center, Philippines

10.1136/gutjnl-2020-IDDF.50

Background Gastrointestinal tuberculosis (GITB) remains to be a major health problem in developing countries and results in significant morbidity and mortality. It can present with diagnostic challenges, and a high index of suspicion should be considered specifically in endemic regions. Clinical, radiographic, endoscopic, and histopathologic findings complement one another in establishing the diagnosis. It may involve any part of the gastrointestinal tract. Isolated colonic tuberculosis is less common. The hypertrophic type resembles an inflammatory mass that mimics malignant neoplasm, which can cause intestinal obstruction. It is important to recognize GITB early to prevent complications and the possible need for surgery. We report a hypertrophic type of isolated cecal tuberculosis mimicking colonic malignancy.

Methods A 56y/o male with chronic renal insufficiency presented with intermittent, colicky abdominal pain associated

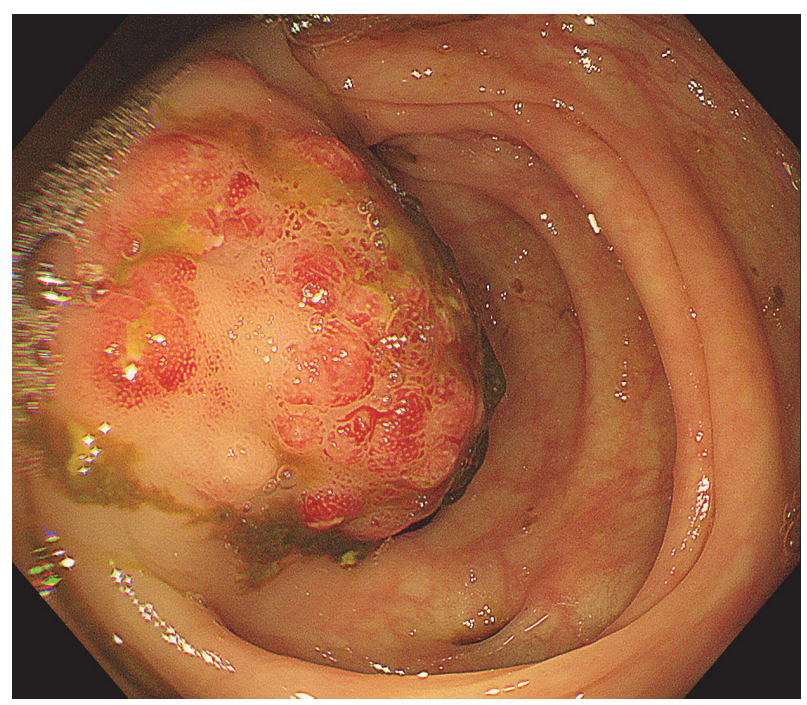

Abstract IDDF2020-ABS-0042 Figure 1 Cecal mass almost completely obstructing the lumen with changes in bowel movement and weight loss. He presented with fever, a slightly distended abdomen with hyperactive bowel sounds.

Results Complete blood count revealed anemia (Hgb 9.9 g/ $\mathrm{dL})$, leukocytosis $\left(17.3 \times 10^{9} / \mathrm{L}\right)$ and elevated serum creatinine (1166 umol/L). Abdominal CT scan showed ascending colon wall thickening with associated pericecal fat stranding and luminal narrowing. Colonoscopy showed a large mass at the cecum near the ileocecal valve.

Histopathology showed chronic granulomatous inflammation with Langhans giant cells and necrosis consistent with cecal tuberculosis.

Anti-TB treatment for category 1 extra-pulmonary TB consists of two (2) months of isoniazid, rifampicin, pyrazinamide, and ethambutol (2HRZE) as intensive phase followed by four (4) months of isoniazid and rifampicin (4HR) as continuation phase.

Conclusions Differential diagnosis of gastrointestinal TB should always be considered in colonic masses producing obstruction among patients in TB-endemic countries. (Figure 1).

\section{IDDF2020-ABS-0043 INTERVAL TIME BETWEEN GASTRIC PREPARATION AND MAGNETIC- CONTROLLED CAPSULE ENDOSCOPY: A RANDOMIZED CONTROLLED TRIAL}

Jia-chuan Wu*, Rui-yan Chen, Xiao-dong Chen, Li-fang Ye, Xiao-qiao Yang, Biao Liang, Hai-zhu Li, Li-li Ye. Guangdong Second Provincial General Hospital, China

\subsection{6/gutjnl-2020-IDDF.51}

Background To investigate the best interval time between gastric preparation and magnetic-controlled capsule endoscopy by comparing the effect of various interval time on image quality of stomach, safety, tolerance and positive lesions through a randomized controlled trial.

Methods 80 patients referred for MCE from Jan. 2018 to Dec. 2018 were randomly assigned to three groups, interval time with 30 minute and less (A), 30 to 60 minutes (B) or 60 minutes and more (C). All patients fasted for at least 8 hours before MCE and administrated with dimethicone, pronase and sodium bicarbonate. Cleanliness and visualization scores, safety, tolerability, additional water required and diagnostic sensitivity were compared. A multi-factor analysis of cleanliness and visualization scores was conducted.

Results The total cleanliness scores were (mean \pm SD) 20.44 \pm 2.61 (A), $20.96 \pm 1.68$ (B), and $20.56 \pm 2.90$ (C). The total visualization scores (mean $\pm \mathrm{SD}$ ) were $16.33 \pm 2.01$ (A), 16.41 \pm 1.50 (B), and $16.07 \pm 1.96$ (C). There was no statistical difference among groups $\mathrm{A}, \mathrm{B}$ and $\mathrm{C}(\mathrm{P}>0.05)$. MCE detected positive findings in $16(59.3 \%), 18(66.7 \%)$ and $15(55.6 \%)$ patients in group $\mathrm{A}, \mathrm{B}$ and $\mathrm{C}$ respectively, with no significant difference between groups $(\mathrm{P}>0.05)$. Additional water required during MCE were (mean $\pm \mathrm{SD})$ 107.41 $\pm 70.31 \mathrm{~mL}(\mathrm{~A}), 46.30$ $\pm 39.04 \mathrm{~mL}(\mathrm{~B}), 79.63 \pm 65.43 \mathrm{~mL}(\mathrm{C})$, and it was much less in group $\mathrm{B}$ than the other two groups $(\mathrm{P}=0.003)$. There was a negative correlation between cleanliness and visualization scores and the infection of $\mathrm{H}$. pylori $\left(\mathrm{r}_{\mathrm{s}}=-0.326, \mathrm{P}=0.003\right.$ and $\left.r_{s}=-0.235, P=0.035\right)$.

Conclusions Patients administrated with dimethicone, pronase and sodium bicarbonate 30 minute and less, 30 to 60 minutes, 60 minutes and more were not statistically different 
in cleanliness, visualization and positive rates. But groups of 30 to 60 minutes got the least supplement of water during MCE, and it may be the best interval time between gastric preparation and MCE.

\section{IDDF2020-ABS-0044 TO EVALUATE THE FEASIBILITY AND EFFICACY OF NON-RADIOLOGICAL TEST TO DETECT POST OPERATIVE LEAK AFTER BARIATRIC SURGERY}

Nikhil Gupta*, Shubham Goel, Arun K Gupta, ASN Rao. ABVIMS Dr RML Hospital, India

\subsection{6/gutjnl-2020-IDDF.52}

Background Gastrograffin study under fluoroscopic guidance is a norm after sleeve gastrectomy in most of the bariatric centres to detect the post operative leak. Shifting the patient to fluoroscopic room on post operative day one and radiation exposure are major concerns associated with this protocol. A lot of studies are available to prove the efficacy of bed side oral methylene blue test to detect anastomotic leak after esophagojejunal anastomosis. We used the same principle in our bariatric patients.

Methods This prospective, interventional study was conducted on 23 patients undergoing sleeve gastrectomy for morbid obesity at our institution.

Results All patients were haemodynamically stable on post operative day-1. There was no evidence of methylene blue in the drain in any of the patients. All patients were started oral liquids after the test.

Conclusions Routine gastrograffin study under fluoroscopic guidance in the post operative period to detect leak following sleeve gastrectomy is a cumbersome procedure and can be avoided. Oral bed side Methylene blue test is a good alternative.

\section{IDDF2020-ABS-0045 CLINICAL SIGNIFICANCE OF INTRA- ABDOMINAL PRESSURE MEASUREMENT IN PATIENTS WITH ACUTE ABDOMEN REQUIRING EXPLORATORY LAPAROTOMY}

${ }^{1}$ Binita Goswami*, ${ }^{2}$ Sumit Pathania, ${ }^{2}$ Arun K Gupta, ${ }^{2}$ Nikhil Gupta. ${ }^{1}$ Maulana Azad Medical College, Delhi, India; ${ }^{2} A B V I M S$ Dr RML Hospital, India

\subsection{6/gutjnl-2020-IDDF.53}

Background Intra-abdominal Hypertension (IAH) has been identified as a significant risk factor for morbidity and mortality. Our study was done to monitor intra-abdominal pressure (IAP) in patients with acute abdomen requiring exploratory laparotomy and to determine its effect on multiple organs by measuring SOFA score and its effect on the outcome of patients.

Methods It was an observational study in which IAP was measured pre and post-operatively by inserting a catheter in the urinary bladder using manometer 6 hourly in patients of acute abdomen requiring exploratory laparotomy.

Results Of 60 patients 23 (38\%) had IAP (mean) and 24 (40\%) had IAP (max) more than $12 \mathrm{mmHg}$. Patients with intestinal obstruction and intestinal perforation with peritonitis had an incidence of raised IAP. There was an increase in hospital stay in patients with IAH, and this was statistically significant $(p<0.05)$. There was positive correlation coefficient relationship between IAP (mean) and (max) with hospital stay $r \operatorname{IAP}(\max )=0.4757$ and $\mathrm{r} \operatorname{IAP}(\max )=0.4893$. Elevated IAP affects all organs, but cardiovascular, renal and respiratory systems were more prone to failure, and there was a positive correlation between IAP and organ failure.

Conclusions IAP should be routinely measured in patients of acute abdomen requiring exploratory laparotomy. Patients with raised IAP preoperatively should be taken up for emergency surgery as soon as possible for a better outcome before deleterious effects of raised IAP on various organ system set in.

\section{IDDF2020-ABS-0048 STUDY ON THE CHANGES OF COAGULATION INDEXES IN PATIENTS WITH CROHN'S DISEASE}

Yi Yu*. Department of Critical Care Medicine, The Second Affiliated Hospital of Guangzhou University of Chinese Medicine, China

\subsection{6/gutjnl-2020-IDDF.54}

Background Thromboembolism is a life-threatening complication of Crohn's disease(CD), CD patients were four times more likely to develop thromboembolism than healthy people, its relative risk increased to 15 times during the active period of disease. To investigate the association between the changes of coagulation indexes in patients with CD.

Methods The clinical data of $78 \mathrm{CD}$ patients who diagnosed for the first time (CD group) and 75 health checkup (control group) from June 2010 to July 2018 in the MIMIC-III database were analyzed retrospectively. The coagulation indexes of the two groups and those of patients with different activity in the CD group were compared, the correlation between coagulation indexes and Crohn's disease activity index (CDAI) scores of Crohn's disease were analyzed.

Results The levels of fibrinogen, platelet count and PT in the CD group were significantly higher than those in the healthy control group $(\mathrm{P}<0.05)$. The levels of fibrinogen, FDP, and APTT in patients with remission stage were significantly lower than those in patients with moderate activity stage $(\mathrm{P}<0.05)$. Pearson linear correlation analysis showed that fibrinogen, platelet count, FDP and APTT were positively correlated with CDAI $(\mathrm{P}<0.05)$.

Conclusions The coagulation indexes of CD patients are significantly higher than those of normal people, and fibrinogen, platelet count, FDP and APTT are closely related to the activity of Crohn's disease.

\section{IDDF2020-ABS-0049 APPLICATION OF NUTRITIONAL RISK SCREENING AND BMI IN NUTRITIONAL ASSESSMENT OF PATIENTS WITH CROHN'S DISEASE}

Yi Yu*. Department of Critical Care Medicine, The Second Affiliated Hospital of Guangzhou University of Chinese Medicine, China

\subsection{6/gutjnl-2020-IDDF.55}

Background To investigate the association between the nutritional risk screening (NRS-2002) and body mass index (BMI) were used to assess the nutritional status of patients with Crohn's disease.

Methods 134 patients with Crohn's disease admitted to the First Affiliated Hospital of Sun Yat-sen University from June 